\title{
The Effects of Nonconcurrent and Concurrent Relaxation Training on Cardiovascular Reactivity to a Psychological Stressor
}

\author{
Patricia A. Cole ${ }^{1,4}$ Cynthia S. Pomerleau, ${ }^{2}$ and Janel K. Harris ${ }^{3}$ \\ Accepted for publication: October 21, 1991
}

Eight patients in a cardiac rehabilitation program, after exposure to two psychological stressors approximately equivalent with respect to cardiovascular reactivity, were given nonconcurrent progressive muscle relaxation training and retested for reactivity. They were then provided with relaxation training concurrently with one of the stressors and exposed again to the two stressors. No significant effects for nonconcurrent progressive muscle relaxation training were detected. Concurrent training, in contrast, produced reductions in both systolic and diastolic blood pressure. Reductions resulting from training on the target stressor showed little tendency to generalize to the nontarget stressor; the discrimination was particularly well defined for systolic blood pressure. We conclude that muscle relaxation techniques are maximally effective in reducing reactivity to psychological stressors when relaxation training is provided concurrently with the stressor. Our findings further suggest that to inculcate the relaxation response reliably across different situations, specific training to enhance generalization may be needed.

KEY WORDS: cardiovascular reactivity; concurrent relaxation training; nonconcurrent relaxation training; stress.

\footnotetext{
${ }^{1}$ Departments of Anesthesiology and Psychology and Social Sciences, Rush Pain Center, Rush Presbyterian St. Luke's Medical Center, Room 110 Professional Building, 1725 West Harrison Street, Chicago, Illinois 60612.

${ }^{2}$ Behavioral Medicine Program, University of Michigan, Department of Psychiatry, Ann Arbor, Michigan 48109.

${ }^{3}$ Division of Epidemiology, University of Minnesota School of Public Health, Minneapolis, Minnesota 55455.

To whom correspondence should be addressed.
} 


\section{INTRODUCTION}

Most studies of relaxation training as a technique for dampening cardiovascular reactivity have involved training in a quiet, nonstressful environment, followed by exposure to a stressor with instructions to emit the response learned in the quiet environment. A number of investigators have suggested that the mixed results of these studies may in fact be attributable to a failure of the training to generalize to the stressful environment and have proposed that training should take place under actual exposure to stressors, either from the outset or after initial training under nondistracting conditions (Harris et al., 1984). DeGood and Adams (1976), for example, found two types of concurrent training (heart rate biofeedback training and progressive muscle relaxation) to be significantly more effective than a control condition in reducing heart rate reactivity. Subsequent studies (Steptoe, 1978; Steptoe and Ross, 1982; Harris et al., 1984) have supported and extended this finding using a variety of cardiovascular measures as dependent variables. The only study directly comparing concurrent vs. nonconcurrent training appeared to show superior results for concurrent training, although the differences were not significant (Kirsh and Henry, 1979).

Despite these promising observations, the therapeutic utility of concurrent relaxation training is clearly limited unless training with one stressor generalizes to other environments. Steptoe and Ross (1982) reported that after concurrent training with three stressors, treatment groups generalized to a fourth, untrained, stressor. These results have not been replicated, however, nor have less extensive training regimens been tested. The present study involved a repeated-measures design with eight patients in a cardiovascular rehabilitation program. After exposure to two stressors (intended to be approximately equivalent with respect to cardiovascular reactivity), patients were given nonconcurrent progressive muscle relaxation training and retested for reactivity; they were then provided with relaxation training concurrently with one of the two stressors (four subjects assigned to each of the two target stressors) and exposed again to the two stressors. The objectives were to determine (1) whether nonconcurrent training would produce a significant reduction in representative measures of sympathetic arousal; (2) whether concurrent training would produce further improvement on the trained stressor, over and above nonconcurrent relaxation training; and (3) whether reduced reactivity to the trained stressor would generalize to the untrained stressor. 


\section{METHODS}

\section{Subjects}

Subjects were eight patients (seven males, one female; 46-67 years old) in a cardiac rehabilitation unit who volunteered in response to verbal announcements. All were white, were either employed or retired, and had a high-school education or above. All had been referred for post-myocardial infarction, essential hypertension, and/or coronary artery disease, but were no longer considered at high risk for infarction. Three subjects were taking antihypertensive agents (one diuretic, two beta-blockers). All subjects provided written informed consent and agreed to make no changes in diet, exercise, medication, or smoking behavior for the duration of the study unless ordered to do so by their physician.

\section{Apparatus}

Frontal electromyographic (EMG) activity to provide muscle tension feedback training was obtained using a J\&J EMG Unit (Model M-52) and silver/silver chloride active electrodes, covered with conducting gel, attached to the frontal area $1 \mathrm{~cm}$ above the center of the eyebrows. Systolic and diastolic blood pressure (SBP, DBP) and heart rate (HR) were measured during the probes using an Astropulse Tm blood pressure unit (Model 69) with an automatically inflatable cuff (attached to the subject's nondominant arm) and a digital readout. A Commodore 20 home computer was used for presentation and collection of responses for the mental arithmetic stressor.

\section{Relaxation Training}

Nonconcurrent Relaxation Training. With monitoring equipment attached, subjects were exposed to cue-controlled progressive muscle relaxation training, as described by Bernstein and Borkovec (1973), using tape-recorded instructions. Training continued until subjects could scan their bodies for muscular tension and relax on cue within 1-2 min; the relaxation response was defined as a decrease in frontal EMG to a level of $\leq 6 \mu \mathrm{V}$. Subjects were requested to practice these exercises at home, using a copy of the taped instructions.

Concurrent Relaxation Training. Concurrent training involved exposure to the stressor in a graduated manner, starting with $30 \mathrm{sec}$ of the task, and 
were given a global evaluation of their success in relaxing ("Good" or "Relax a little more," based on EMG) after each trial. Only when physiological measures of reactivity during the task were within $50 \%$ of the subject's relaxation levels at the end of nonconcurrent training was he/she exposed to a slightly longer duration of the stressor.

\section{Stressors}

The mental arithmetic task involved performance of time-limited arithmetic problems on the personal computer, as described by Steptoe (1978); various aspects of the subjects' performance were measured to ensure that individual difficulty levels were kept more or less constant. The role-play stressor included both individualized and standardized role-play situations; the experiment briefly described an uncomfortable situation and then asked the subject to imagine that he/she was in that situation, with trained confederates playing supporting roles. Exposure to each stressor lasted for $3 \mathrm{~min}$.

\section{Physiological Measures}

SPB, DBP, and HR were measured at 5-min intervals during a 20-min adaptation period at the start of each session; the final reading was used as the resting measure. Stress measures were obtained during the second minute of the 3-min stressor. The difference between these readings was used as the measure of reactivity.

\section{Procedure}

Probe sessions were held at intervals of approximately 3-5 days. Following a baseline probe of reactivity, all subjects underwent $8-12$ sessions of nonconcurrent relaxation training (i.e., under standard quiet conditions), as described above. During the second probe session, subjects were instructed to relax during the mental arithmetic and role-play stressors by scanning their bodies for muscular tension and cuing themselves to relax. No feedback of any kind was given.

Subjects then received four to eight sessions of concurrent training as described above in association with one of the two stressors-four with mental arithmetic and four with role playing. [Subjects assigned to the two target stressor groups were matched for behavioral typology as measured by the Jenkins Activity Survey (Jenkins et al., 1979) to control for the possible impact of the Type A behavior pattern upon reactivity.] During the 
third probe session, subjects were instructed to relax during both the mental arithmetic and the role-play stressors. As with Probe 2, no feedback was given.

\section{Data Analysis}

All analyses were carried out using BMDP-2V statistical software for repeated measures designs. Because of the nature of the design (a weak intervention between Probe 1 and Probe 2; between-group experimental manipulations preceding only one of the three probes), nonsignificant overall $F$ values were expected, and a series of nonorthogonal comparisons using the $t$ test for differences among several means (Bruning and Kintz, 1987) was planned: (1) scores on Probe 1 (mean for the two stressors) were compared with corresponding scores on Probe 2 to determine whether nonconcurrent training would produce a significant reduction in reactivity; (2) scores on the target stressor at Probe 3 were compared with comparable scores on Probes 1 and 2 to determine the effectiveness of concurrent training - as a further check, a similar series of comparisons was conducted on the nontarget stressor to ensure that observed effects were not simply a function of time or habituation; and (3) scores on the target stressor at Probe 3 were compared with scores on the nontarget stressor to determine whether reduced reactivity to the trained stressor would generalize to the untrained stressor.

\section{RESULTS}

Reactivity data for the one female and for the three subjects on antihypertensive agents were within a standard deviation of the group norm.

For the first two probes, all subjects were exposed to identical conditions. Therefore, to test the assumption that the two stressors were equivalently reactive and to determine whether there were any fortuitous differences in the pretraining response of the two target stressor groups to either of the stressors, a preliminary factorial ANOVA was conducted using BMDP-2V, with Probe (1 vs. 2 ) and stressor (mental arithmetic vs. role play) as repeated measures and Probe 3 assignment (training on mental arithmetic vs. training on role playing) as a between-group factor. Although role playing was consistently slightly more reactive than mental arithmetic, the differences did not prove to be significant. No significant main or interaction effects were detected between the two Probe 3 groups. No significant differences were detected between Probe 1 and Probe 2. 


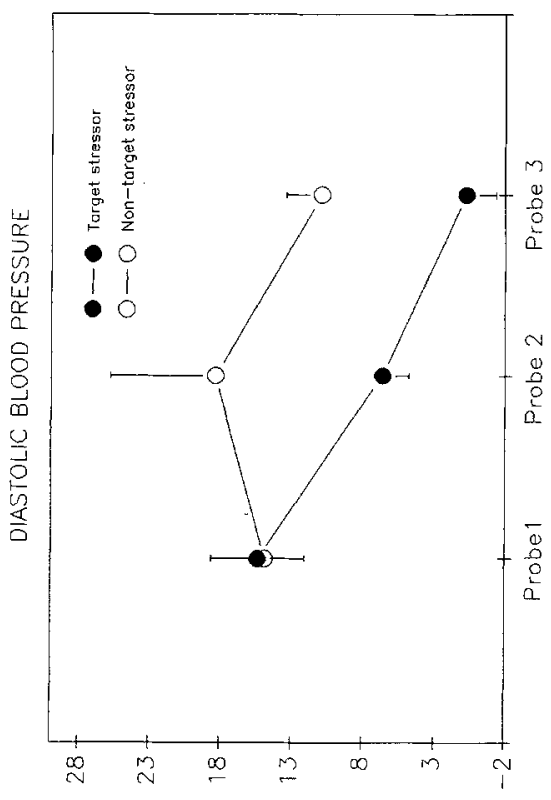

(6 нwu) da0 u! ә Бир4ว

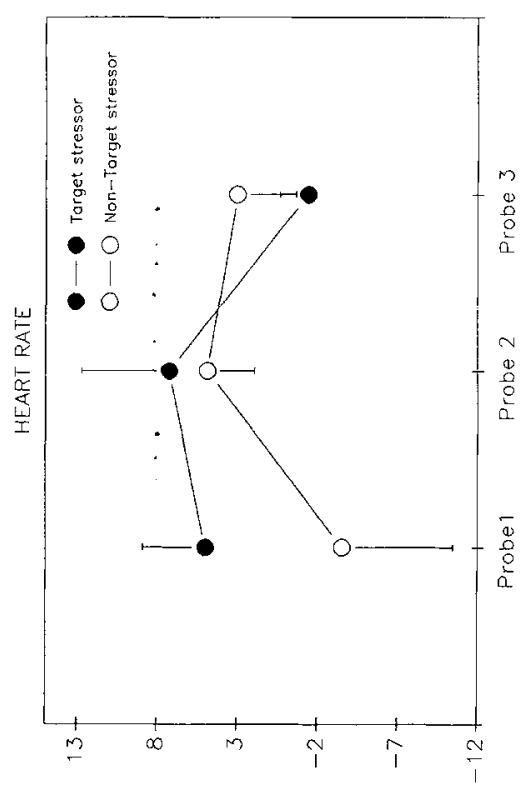

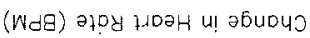
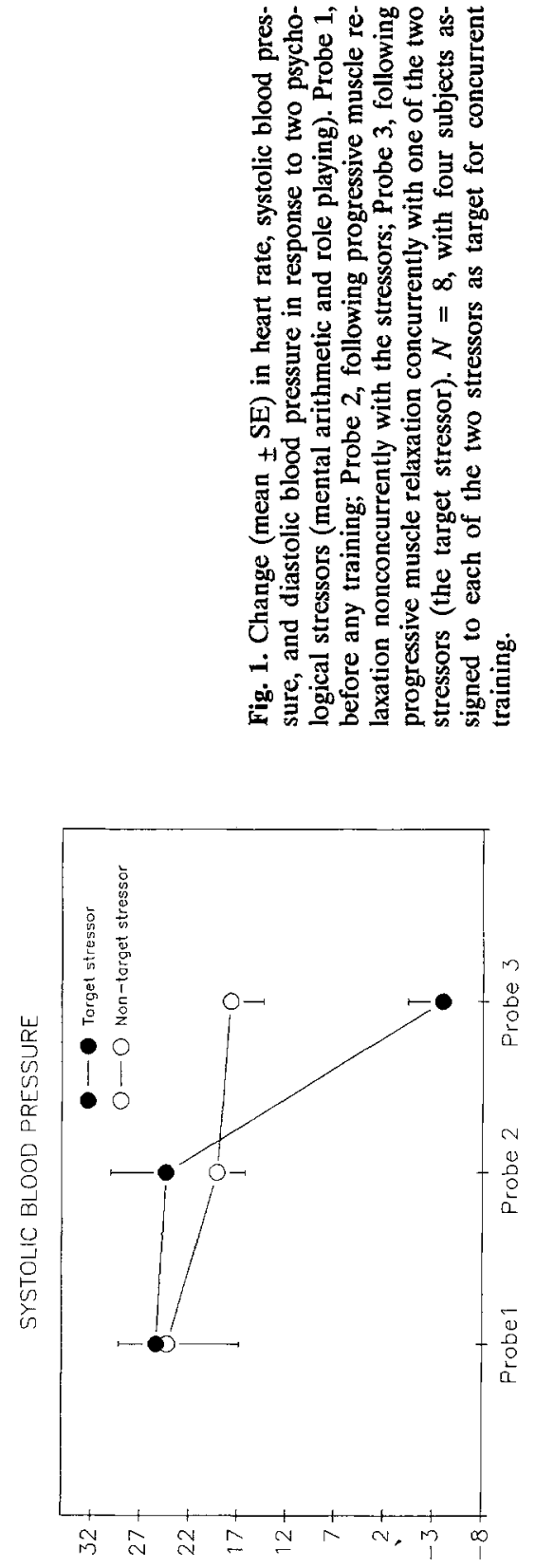

(BHWس) das u! 200040 
Mean scores for the target vs. the nontarget stressor for each of the three probes are shown in Fig. 1. The main data analytic strategy involved a 3 (Probe: 1, 2, and 3) $\times 2$ (training status: target stressor vs. non-target stressor) $\times 2$ (Probe 3 assignment: training during mental arithmetic vs. training during role playing) repeated-measures ANOVA. Since no significant main or interaction effects were detected between the two Probe 3 groups, the analysis was rerun omitting the grouping factor to facilitate further significance testing. No significant differences were detected for heart rate or DBP; for SBP, significant probe $[F(2,14)=10.26 ; p=.0018]$ and probe $\times$ target $[F(2,14)=8.26, p=.0043]$ emerged.

The planned nonorthogonal comparisons yielded the following results. (1) No significant effects were detected for Probe 1 (baseline) vs. Probe 2 (post-nonconcurrent relaxation training). (2) Target stressor scores at Probe 3 (post-concurrent training) were significantly lower than at Probe 1 (baseline) for systolic blood pressure $(p<.001)$ and for diastolic blood pressure $(p<.05)$ but not for heart rate. Systolic blood pressure scores at Probe 3 were also significantly lower than at Probe 2 (post-nonconcurrent training). No significant differences were discovered among probes for the nontarget stressor. (3) Although scores for the nontarget stressor dropped slightly (though not significantly) from Probe 2 to Probe 3 , reactivity to the target stressor at Probe 3 was significantly lower than reactivity to the nontarget stressor for systolic blood pressure $(p<.001)$; similar effects for diastolic blood pressure showed a trend toward significance $(.10>p>.05)$.

\section{DISCUSSION}

No significant effects for nonconcurrent progressive muscle relaxation training were detected in either the preliminary or the main analysis; this observation must be regarded with caution, however, since our failure to detect effects may be attributable to the small sample size and resulting low power. Concurrent training, in contrast, appeared to produce reductions in both systolic and diastolic blood pressure; a definitive test of this inference, however, would require the inclusion of a group given repeated exposure to a stressor between Probe 2 and Probe 3, in order to rule out the possibility that the observed effects were due to adaptation to the stressor. The effects of training on the target stressor showed little tendency to generalize to the nontarget stressor; the discrimination was particularly well defined for systolic blood pressure, as indicated by a significant difference between scores for the target stressor at Probe 3 and comparable scores on the nontarget stressor. 
We conclude that muscle relaxation techniques are probably useful in reducing heightened sympathetic arousal to psychological stressors. Maximal benefit seems to occur when relaxation training is provided concurrently with a stressor. Under the conditions of the present experiment, the relaxation response showed a discrimination, occurring to the psychological stressor for which training had been provided, but not to the stressor for which no training was given. Thus, our findings suggest that to inculcate the relaxation response reliably across situations/stressors, specific training to enhance generalization will be needed [as may have occurred in the study by Steptoe and Ross (1982), who trained relaxation to multiple stressors before introducing an untrained stressor].

\section{ACKNOWLEDGMENTS}

This research was conducted in partial fulfillment of the requirements for a doctoral degree in psychology at Western Michigan University (first author). The authors wish to thank Morton Brown, Ph.D., R. Wayne Fuqua, Ph.D., Frederick Gault, Ph.D., Michael F. Macken, M.D., Ovide F. Pomerleau, Ph.D., and M. Anthony Schork, Ph.D., for advice and encouragement at various stages in the preparation of the manuscript.

\section{REFERENCES}

Bernstein, D., and Borkovec, T. (1973). Progressive Relaxation Training: A Manual for the Helping Professions, Research Press, Champaign, IL.

Bruning, J. L., and Kintz, B. L. (1987). Computational Handbook of Statistics. Scott, Foresman, Glenview, IL.

DeGood, D., and Adams A. (1976). Control of cardiac responses under aversive stimulation. Biofeed. Self-regulat. 1: 373-385.

Harris, J. K., Maldonado, A. J., Fuqua, R. W., Cole, P. A., Gault, F., Garman, F., and Macken, M. S. (1984). Psychological effects of relaxation training during stress in Type A and Type B coronary patients. Presented at the 5th Annual Convention of the Society of Behavioral Medicine, Philadelphia, PA, May.

Jenkins, D., Zyzanski, S., and Rosenman, R. (1979). Jenkins Activity Survey: JAS Manual, Psychological Corp., New York.

Kirsh, I., and Henry, D. (1979). Self-desensitization and meditation in the reduction of public speaking anxiety. J. Consult. Clin. Psychol. 47: 536-541.

Steptoe, A. (1978). The regulation of blood pressure reactions to taxing conditions using pulse transit-time feedback and relaxation. Psychophysiology 15(5): 429-438.

Steptoe, A., and Ross, A. (1982). Voluntary control of cardiovascular reactions to demanding tasks. Biofeed. Self-regulat. 7: 149-167. 